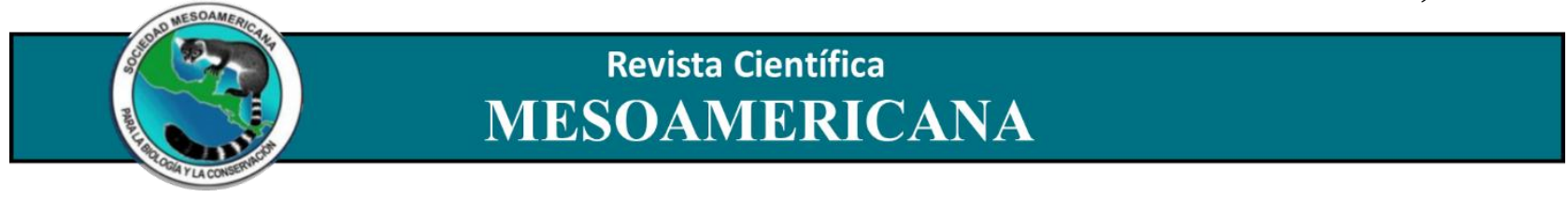

\title{
Potencial regenerativo de bosques de galería en base a diversidad y abundancia de mamíferos en la Reserva Forestal El Montuoso y afluentes del río La Villa, Herrera, Panamá
}

\author{
Pedro G. Méndez-Carvajal ${ }^{12}$ (D) , Iris Gómez de Huertas ${ }^{13}$ (D) Karol M. Gutiérrez- \\ Pineda $^{1}$ (D), Ricardo S. Moreno 4 (iD), Marcos A. Peñafiel ${ }^{1}$ (i) , Amy S. Girón-Rengifo ${ }^{1}$ (iD, \\ Elena Méndez-Carvajal ${ }^{1}$ (D), Pedro A. González-Hernández ${ }^{13}$ (D) \& Angélica Ortiz ${ }^{13}$ iD \\ 1Fundación Pro-Conservación de los Primates Panameños (FCPP), mendezp@fcprimatespanama.org \\ 2Universidad de Panamá, Escuela de Biología, Departamento de Fisiología y Comportamiento Animal, \\ República de Panamá, pedro.mendezc@up.ac.pa \\ 3Universidad de Panamá, Centro Regional Universitario de Coclé (CRU-Coclé), Departamento de Biología, \\ Penonomé, provincia de Coclé, igomez@fcprimatespanama.org \\ 4Fundación Yaguará Panamá, Ciudad del Saber, Edificio 181. rmorenopan@gmail.com
}

Fecha de Recepción: 22-IV-2019. Fecha de Aceptación: 20-VI-2020. Fecha de Publicación: 25-VI-2020.

\section{RESUMEN}

La Reserva Forestal El Montuoso (RFEM) es un macizo boscoso noroccidental de Azuero, protege el nacimiento del río La Villa, fuente hídrica importante. Estudiamos la biodiversidad de mamíferos y el estado poblacional de los monos aulladores endémicos y En Peligro Crítico A.c. trabeata, y su relación con otras especies. Se consideró $A$. c. trabeata como indicador para evaluar su papel como dispersor de semillas para mantener la conexión boscosa en los afluentes al río La Villa. Encontramos una baja densidad poblacional (0.004 ind $/ \mathrm{km}^{2}$ ) respecto a datos anteriores. Reportamos cinco especies de mamíferos no antes detectados en la RFEM. La ausencia de grupos taxonómicos como cérvidos y felinos grandes, mientras los murciélagos filostómidos prevalecen como potencial de dispersión de frutos y semillas para mantener a la reserva como hábitat donador a sus afluentes. La conservación de estas áreas depende de la reforestación con especies de árboles nativos y controlar de forma más enérgica los delitos ambientales de tala y cacería dentro de la reserva. FCPP implementó actividades de entrenamiento, reforestación y distribución de material didáctico, para el área de la RFEM, Chupá y Macaracas.

Palabras Clave: Reserva Forestal El Montuoso, río La Villa, mamíferos, Panamá.

Regenerative potential of gallery forests based on diversity and abundance of mammals in the El Montuoso Forest Reserve and tributaries of the La Villa river, Herrera, Panama

\section{ABSTRACT}

The El Montuoso Forest Reserve (EMFR) is a northwestern forested mountain chain of Azuero, origin of La Villa River, an important water source. We studied the mammal biodiversity and the population status of the endemic and Critically Endangered Azuero howler monkeys A. c. trabeata, and its relationship with other species. We took A.c. trabeata as an indicator to evaluate its role as seed 
dispersers to keep forest connectivity along the tributaries of the La Villa river. We found the population density $\left(0.004 \mathrm{ind} / \mathrm{km}^{2}\right)$ was lower than past records. We found five species of mammals that have not been detected before at the EMFR. We noted absence of taxonomic groups such as cervids and large felines, and prevalence of phyllostomids, as important seed and fruit's dispersers, turning the reserve as the main habitat donor to their tributaries. Conservation in this area depends on reforesting with native tree species and the enforcement of environmental and prosecution for illegal hunting and logging within the reserve. FCPP implemented training activities, reforestation, and distribution of educational materials for the area of RFEM, Chupá, and Macaracas.

Keywords: El Montuoso Forest Reserve, La Villa river, mammals, Panama.

\section{INTRODUCCIÓN}

Los mamíferos son considerados como promotores de la conectividad boscosa por ser dispersores de semillas y polinizadores de flores, mantienen gran parte de la cobertura de los ríos (Rumiz, 2010). Los mamíferos herbívoros promueven el crecimiento abundante de especies de árboles colectores de agua a lo largo de los ríos en Mesoamérica (Rylands et al., 2006; Rosales-Meda, 2006; MéndezCarvajal, 2012). Sin embargo, las actividades antropogénicas podrían estar degradando dichos recursos en cantidad y expansión boscosa debido a la agricultura, ganadería extensiva, extracción maderable y el uso de agroquímicos (Arroyo-Rodríguez et al., 2005; Masís et al., 2008). La cantidad de especies y densidad poblacional para mamíferos terrestres y arborícolas podría variar producto de la caza extrema y tala, alterando la dinámica poblacional de animales de sotobosque que cambian su patrón de forrajeo dependiendo de los dispersores de alimentos, arborícolas y voladores (Estrada et al., 2004; Garibaldi et al., 2004; Zunino et al., 2007). Algunos mamíferos podrían ser importantes para actividades de conservación, si detectamos especies como indicadores de estabilidad ecológica, mientras que otros podrían ser considerados como contribuyentes en el proceso de regeneración de los bosques (Cowlishaw \& Dunbar, 2000; Paliza-García, 2018). Se estima que uno de los mamíferos más representativos en los afluentes panameños son los monos aulladores, los mismos están relacionados directamente con los árboles estructurales, medicinales y maderables (e.g. Anacardium excelsum, Bursera simaruba, Cecropia spp.) (Gaulin et al., 1980; Massey, 1987; Méndez-Carvajal, 2005; Estrada et al., 2006; Rosales-Meda, 2006). Especies de monos aulladores también presentan plasticidad ecológica y pueden resistir un poco más la transformación de hábitats naturales por cultivos, Alouatta palliata por ejemplo, se reporta en Los Tuxtlas, México, así también en cultivos de Lanchúa, Guatemala con A. pigra, igualmente poblaciones de A. palliata se reportan en zonas fragmentadas de Guanacaste, Costa Rica, considerados como una especie de demografía medible para garantizar la regeneración en lugares alterados (Estrada et al., 2006). Al ser modificadores de estructuras vegetales, los monos son "ingenieros ecológicos" y su paso por las ramas dejan influencia marcada en el patrón de movimiento de animales de sotobosque, depredadores y estructuras de bosque (Jones et al., 1994; Boogert et al., 2006). Este papel ecológico de los monos aulladores se podría relacionar con alto índice de diversidad de plantas y animales, por lo que planteamos visualizar la presencia del mono aullador y otras especies como indicadoras de alto potencial de regeneración y conectividad boscosa en los afluentes del río La Villa. El río La Villa, es una de las cuencas hidrográficas más importantes de Panamá, nace en las faldas del macizo montañoso de la Reserva Forestal El Montuoso (RFEM) (Méndez-Carvajal et al., 2004). La reserva fue declarada protegida por Ley 12 del 15 de marzo de 1977 y en 2004 se evalúo formalmente su mastofauna. El estudio liderado por JICA-ICAB-Universidad de Panamá realizó un muestreo de mamíferos reportando ocho Órdenes, 16 Familias con 38 Especies de mamíferos, siendo ocho especies arborícolas estrictas (Méndez-Carvajal et al., 2004). Estudios previos en 2005 y 2006 evaluaron por 
primera vez la población de Alouatta coibensis trabeata para las zonas bajas de la provincia de Herrera (Méndez-Carvajal, 2005; 2006) y en la última revisión se incluyó las tierras altas de RFEM donde se encontró a $A$. c. trabeata con una población de solo 37 individuos divididos en seis grupos con promedio de 6.1 individuos/grupo, una densidad de 0.5 individuos $/ \mathrm{km}^{2}$ y 0.08 grupos $/ \mathrm{km}^{2}$ en un área de estudio de $69.83 \mathrm{~km}^{2}$ (Méndez-Carvajal, 2013). Una población ausente y extirpada del mono araña de Azuero Ateles geoffroyi azuerensis se reportó en el mismo estudio bajo el mismo esfuerzo de muestreo de 360 horas (Méndez-Carvajal, 2013). La ausencia de este último primate también figura en la lista como la especie de primate no humano más amenazada del país con solo 150 individuos calculados en vida silvestre (Méndez-Carvajal y Ruiz-Bernard, 2009; Méndez-Carvajal, 2013). En 2017 la Fundación Pro-Conservación de los Primates Panameños (FCPP) firmó un convenio de cooperación con el Ministerio de Ambiente de Panamá (Mi Ambiente) No. CAF-003-2017, y se incorporó a las organizaciones no gubernamentales que lideran proyectos de conservación dentro del Programa de Inversión para la Restauración de Cuencas Hidrográficas Prioritarias (PROCUENCAS). Se emprendió el Proyecto de Monitoreo de Primates y Fauna Relacionada al río la Villa y afluentes, en un esfuerzo comparativo de la población de A. c. trabeata y otros mamíferos arborícolas vs terrestres, en la cabecera del río La Villa y una evaluación de la diversidad de mamíferos en bosques de galería adyacentes tales como río Gato, río Estivaná en el pueblo de Macaracas, provincia de Herrera y Chupá en la provincia de Los Santos. El propósito de esta investigación es reconocer la biodiversidad de mamíferos en los diferentes nichos del bosque, desde arborícola, terrestre y aéreo, para establecer especies que podrían considerarse potenciales indicadores o agentes regeneradores naturales de la vegetación de galería en Azuero. Los bosques de galería mantienen condiciones nutritivas específicas en el suelo facilitando el crecimiento de flora, y acumulación de materia vegetal húmeda que previene la sofocación del sol y la auto-inmisión (CorreaGómez \& Stevensson, 2010). Su preservación ayuda al ciclo hídrico, la calidad del agua y a la protección de los recursos que ampara como fuente de proteína al hombre del campo (RodríguezSosa et al., 2018). Los bosques de galería son excelentes como recurso donador faunístico y florístico a zonas perturbadas, debido a la conectividad que mantienen como transporte acuático y puente biológico con otras zonas boscosas (Garibaldi et al., 2004; da Silva et al., 2008; Méndez-Carvajal, 2012). Siendo RFEM el punto máximo de referencia en diversidad para el río La Villa, nos interesa exponer la riqueza de especies de mamíferos como agente potencial de regeneración y comparar en la actualidad la diversidad encontrada en zonas con alta deforestación para valorar la biodiversidad de mamíferos y sus posibles ascensos/descensos en densidad poblacional por efecto borde como indicador de deterioro antropogénico en primates no humanos y otra fauna relacionada (Garibaldi $e t$ al., 2004; Rodríguez-Sosa et al., 2018).

\section{MATERIALES Y MÉTODOS}

\section{Área de estudio}

Se emplearon dos áreas de muestreo, una fue la zona boscosa nativa de la RFEM (Tres Puntas, Alto del Higo y Caras Pintadas) ubicadas en la comunidad de Chepo de Las Minas, provincia de Herrera, con Bosque Maduro Continuo $\left(0.01 \mathrm{~km}^{2} ; 27 \%\right.$ de la reserva) caracterizado como Perennifolio Ombrófilo Tropical Latifoliado Submontano (Tosi, 1971), reportado para la zona de muestreo Tres Puntas y Caras Pintadas (N 0744' 02.7" y 0 8047'52.2") con árboles de Quercus sp., y Calophyllum sp., mayormente especies de frutos carnosos. Estos hábitats son considerados como Bosque Muy Húmedo Pre-montano con oscilaciones entre 700 a 1,000 metros de altura y pluviosidad de 2,500 mm por año, con una antigüedad estimada de 120 años (Garibaldi et al., 2004). La estación seca aparece de mediados de diciembre a mayo, la lluviosa de mayo a principios de diciembre (Garibaldi et al., 2004). La segunda zona de muestreo se remitió a bosques de galería del pueblo de Macaracas, provincia de Herrera y Los Santos, específicamente los afluentes río Estivaná, Gato y Chupá (N 07²3' 
41.3" y $080^{\circ} 50^{\prime} 35.9^{\prime \prime}$ (Figura 1). Estos se caracterizan como Bosques de Galería y Submontano en cerro Chupá. Árboles característicos de esta zona fueron los Anacardium spp., Spondias spp. y Cecropia spp. A nivel socioeconómico la región de la RFEM se ha caracterizado por suelos pobres, por tanto, prevalece la actividad de ganadería expansiva y siembras de subsistencia, en su mayoría siembras estacionales de arroz, maíz, poroto, frijol de bejuco, otoe y yuca; siembras permanentes de café, piña, pixbae, marañón, guineo, naranja y plátano (Arcia, 2004).

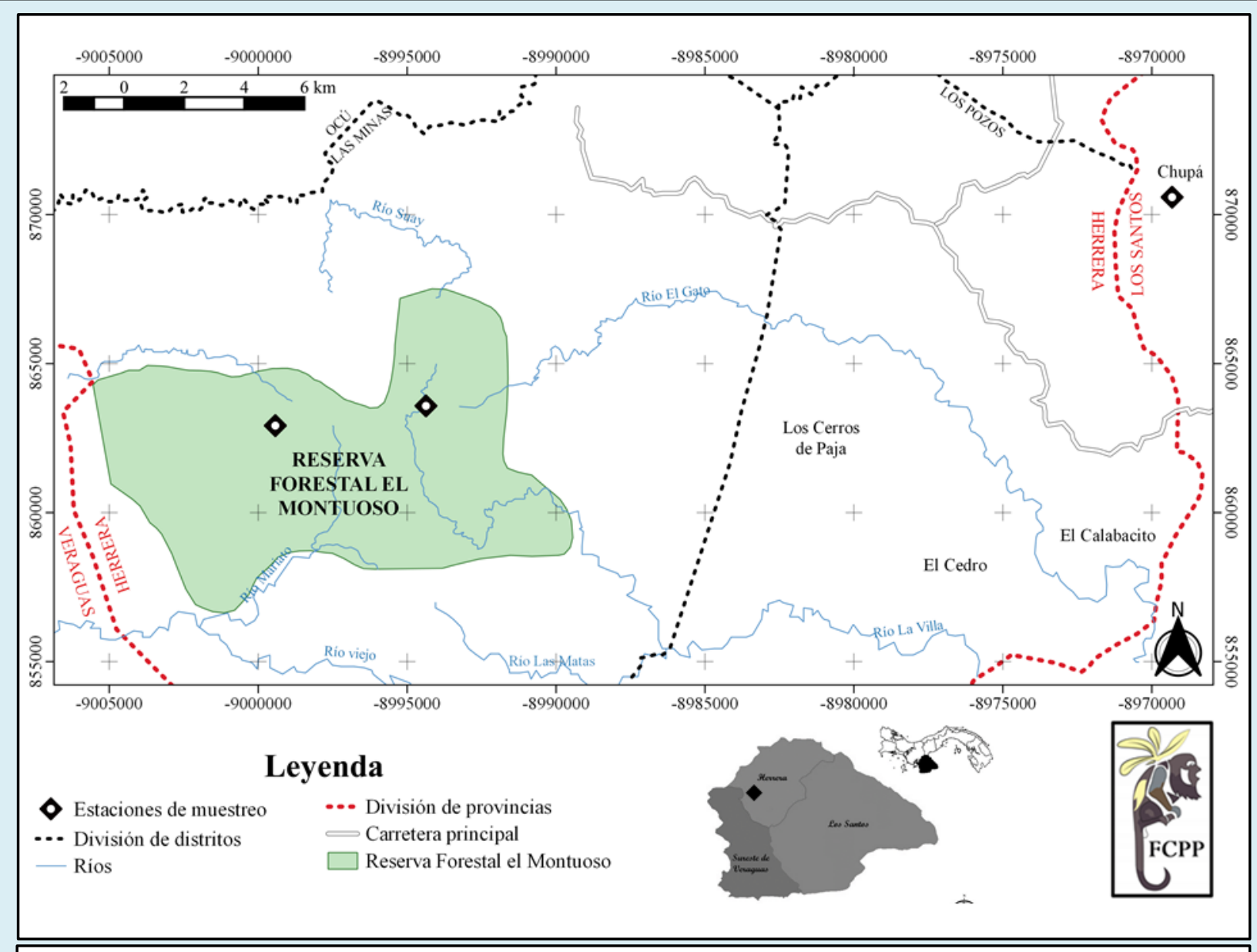

Figura 1. Área de estudio.

\section{Detección de mamíferos}

Se colectó data en tres periodos de muestreos de cinco a 10 días por mes, en estación lluviosa de junio, julio y agosto 2018. Se utilizó el transecto de franja para avistar y cuantificar individuos por área muestreada en la zona boscosa conectada RFEM, utilizando un promedio de ancho de 20 metros de cada lado y un largo de sendero de $1 \mathrm{~km}$ (Ross \& Reeve, 2011). Se empleó binoculares, GPS y brújula para detectar mamíferos de sotobosque y arborícolas en caminatas de 06:00 a 7:00 hrs y de 18:0020:00 o bien 00:00-01:00 hrs. Se recorrió con velocidad de $1 \mathrm{~km} / \mathrm{hr}$ (se anexo dos periodos nocturnos que varió dependiendo de la lluvia). Se anotó nombre científico de especie, posicionamiento global, número de individuos (si fuese grupal), actividad y especie de árbol relacionado cuando pudiera ser identificado. Se colocaron cinco cámaras Bushnell Trophy Cam sin reflector automático de luz, con detector de calor y movimiento. Las cámaras se mantuvieron a un nivel promedio de 12 metros de altura en estrato medio del bosque enfocando la cámara a horquetas y pasaderos para mamíferos 
arborícolas (Méndez-Carvajal, 2014). Dos cámaras fueron colocadas en sotobosque para ambos sitios a altura de un pie del suelo para captar mamíferos terrestres. Se utilizó 2-3 redes de niebla para atrapar murciélagos de sotobosque, la misma posee cuatro bolsas y cubre de 0 a 3 metros de alto y entre 6 a 12 metros de largo, fueron abiertas de 18:00 a 01:00 hrs (Wilson et al., 1996). Se utilizó la clave de roedores de Méndez (1993) y la clave de murciélagos de Handley (1981), se confirmó dudas con ilustraciones e información ecológica provista por Reid (1997). Se colocó 15 trampas Thomahawks y Sherman como complemento para ampliar la detección de mamíferos pequeños como roedores y zarigüeyas (Wilson et al., 1996). Todo animal atrapado fue pasado por clave taxonómica pesado, y medido en sus antebrazos, largo total y cola, luego liberado con el mayor cuidado posible. No hubo método de marcaje, se utilizó convenientemente las cicatrices naturales de los animales para reconocer en lo posible su recaptura y eliminarlo así de los cálculos de número de individuos por especie. Adicional a estos métodos puntuales, se hizo anotaciones Ad-libitum, búsqueda de rastros utilizando la guía de rastros de Aranda (2000). Se realizó conteo de ruta y postes de escucha a libre albedrío (Méndez-Carvajal, 2013).

\section{Análisis estadístico}

Se utilizó la fórmula empleada por Glanz (1992), para cálculo de densidad poblacional en base a N (número de individuos) por dos veces el ancho de la franja por largo total (2LW) ( $D=N / 2 \mathrm{LM})$. En este trabajo se estudia una población "estática" con un margen de error en la medida en que la muestra representa una población sin contar tasa de nacimiento, muerte, movimiento y detección al 100\% de acuerdo a Magurran (2007) y Gotelli y Colwell (2013). Se estableció gráficas de tendencia de actividad circadiana con el programa Past4.02, para esto se tomó en consideración a las especies que tenían más de 11 eventos fotográficos, con intervalos de 30 a más minutos por fotografía si era de una misma especie (Mosquera-Muñoz, 2015). Se realizó Índices de Diversidad Alpha (Dominancia (D), Simpson (1-D), Shannon (H'), Margalef, Equitatibidad_J, Fisher_alpha, Berger-Parker, Chao-1) (Heltshe \& Forrester, 1983; Chao, 1987; Colwell \& Coddington, 1994). Se aplicó Whittaker para determinar la diferencia de especies de murciélagos entre la RFEM y Chupá (Whittaker, 1960).

\section{RESULTADOS}

Se detectó un total de 44 Especies, 37 Géneros, 18 Familias y nueve Órdenes de mamíferos (Cuadro 1). Densidades y estructuras grupales para A.c. trabeata fueron de mayor número en RFEM que en los afluentes como Chupá, mientras que no se detectó primates en Estivaná y río Gato (Cuadro 2). Utilizando las tres densidades se calculó el promedio total de 0.023 grupos $/ \mathrm{km}^{2}$ como densidad general (Cuadro 3). Esta densidad nos refiere más adelante un total de $0.04 \mathrm{ind} / \mathrm{km}^{2} \mathrm{y}$ en total un aproximado de 27 individuos totales para la RFEM contando con un área total de muestreo de 98.83 $\mathrm{km}^{2}$. Con el método de Fototrampeo en el sotobosque se logró establecer una tendencia de la actividad circadiana de cuatro especies (Figura 2).

Los datos obtenidos por los diferentes métodos de muestreos para grupos de mamíferos terrestres y voladores mostraron variaciones en los índices de diversidad dentro de la RFEM. Los mamíferos terrestres presentaron una baja dominancia (0.1687), una diversidad alta según Simpson (1-D), pero con baja riqueza y abundancia. En comparación los murciélagos presentaron un índice de dominancia de 0.3037 , debido a la mayor captura de Carollia perspicillata, baja diversidad, abundancia y riqueza. En Chupá también presentó una dominancia de 0.3967, en este caso por la especie Artibeus jamaicensis, al igual que en la RFEM presentó una baja riqueza y abundancia (Cuadro 4). El Índice de Whittaker muestra un $46.67 \%$ de diferencia entre las especies de murciélagos detectadas en la RFEM y Chupá. Horas de esfuerzo por gira fueron variables debido al clima (Cuadro 5, Figura 4). 
Cuadro 1. Especies de mamíferos detectadas en las RFEM, provincia de Herrera y comparación con la lista de hace 14 años. Se reportan nueve Órdenes, 18 familias, 37 Géneros y 44 especies. En sombra las especies nuevas.

\begin{tabular}{|c|c|c|c|c|}
\hline Orden & Familia & Especie & $\begin{array}{c}\text { Méndez-Carvajal } \\
\text { et al., } 2004\end{array}$ & Este estudio \\
\hline Artiodactyla & $\begin{array}{c}\text { Cervidae } \\
\text { Tayassuidae }\end{array}$ & $\begin{array}{l}\text { Mazama temama } \\
\text { Pecari tajacu }\end{array}$ & $\begin{array}{l}\text { detectado } \\
\text { detectado }\end{array}$ & $\begin{array}{c}\text { no detectado } \\
\text { detectado }\end{array}$ \\
\hline \multirow[t]{9}{*}{ Carnivora } & Felidae & Leopardus wiedii & no detectado & detectado \\
\hline & & Leopardus pardalis & detectado & detectado \\
\hline & & Puma yagouarundi & no detectado & detectado \\
\hline & Mustelidae & Eira barbara & detectado & detectado \\
\hline & & Conepatus semistriatus & detectado & detectado \\
\hline & Procyonidae & Bassaricyon gabbii & detectado & no detectado \\
\hline & & Nasua narica & detectado & detectado \\
\hline & & Potus flavus & detectado & detectado \\
\hline & & Procyon lotor & detectado & no detectado \\
\hline \multirow[t]{16}{*}{ Chiroptera } & Phyllostomidae & Artibeus intermedius & detectado & detectado \\
\hline & & Artibeus jamaicensis & detectado & detectado \\
\hline & & Artibeus lituratus & detectado & detectado \\
\hline & & Artibeus watsoni & detectado & detectado \\
\hline & & Artibeus phaeotis & detectado & detectado \\
\hline & & Carollia castanea & detectado & detectado \\
\hline & & Carollia perspecillata & detectado & detectado \\
\hline & & Chiroderma villosum & detectado & no detectado \\
\hline & & Desmodus rotundus & detectado & detectado \\
\hline & & Mimon crenulatum & detectado & no detectado \\
\hline & & Micronycteris hirsuta & detectado & no detectado \\
\hline & & Myotis nigricans & detectado & no detectado \\
\hline & & Sturnira ludovici & detectado & no detectado \\
\hline & & Uroderma bilobatum & detectado & no detectado \\
\hline & & Vampirops helleri & detectado & no detectado \\
\hline & & Vampyressa pusilla & no detectado & detectado \\
\hline
\end{tabular}




\begin{tabular}{|c|c|c|c|c|}
\hline \multirow{5}{*}{ Didelphimorphia } & & Lyonicteris spurrelli & no detectado & detectado \\
\hline & Thyropteridae & Thyroptera tricolor & detectado & no detectado \\
\hline & Vespertilionidae & Rhogessa tumida & detectado & no detectado \\
\hline & Didelphidae & Didelphis marsupialis & detectado & detectado \\
\hline & & Caluromys derbianus & no detectado & detectado \\
\hline Lagomorpha & Leporidae & Sylvilagus dicei & detectado & no detectado \\
\hline Primates & Cebidae & Cebus imitator & detectado & detectado \\
\hline \multirow{8}{*}{ Rodentia } & Atelidae & Alouatta coibensis & detectado & detectado \\
\hline & Agoutidae & Cuniculus paca & detectado & detectado \\
\hline & & Dasyprocta punctata & detectado & detectado \\
\hline & Cricetidae & Oecomys bicolor & no detectado & detectado \\
\hline & Echimydae & Hoplomys gymnurus & detectado & no detectado \\
\hline & & Proechymis semispinosus & detectado & detectado \\
\hline & Sciuridae & Sciurus granatensis & detectado & detectado \\
\hline & & Sciurus variegatoides & detectado & detectado \\
\hline Pilosa & Myrmecophagidae & Tamandua mexicana & no detectado & detectado \\
\hline Cingulata & Dasypodidae & Dasypus novemncinctus & detectado & detectado \\
\hline
\end{tabular}

Cuadro 2. Presencia y estructura grupal de A.c. trabeata por áreas de estudio.

\begin{tabular}{|cccccccc|}
\hline Grupo & क & $\&$ & $\mathbf{j}$ & $\mathbf{i}$ & $\mathbf{P}$ & $\begin{array}{c}\text { Total } \\
\text { ind/grupo }\end{array}$ & $\begin{array}{c}\text { Sitio de } \\
\text { estudio }\end{array}$ \\
\hline $\mathbf{1}$ & 2 & 3 & 2 & 0 & 1 & 8 & RFEM1 \\
$\mathbf{2}$ & 1 & 2 & 0 & 0 & 0 & 3 & RFEM2 \\
$\mathbf{3}$ & 1 & 2 & 1 & 0 & 0 & 4 & Chupá \\
Total & 4 & 7 & 3 & 0 & 1 & 15 & \\
$\mathbf{x}$ & 1.3 & 2.3 & 1 & 0 & 1 & 5 & \\
$\mathbf{\%}$ & 26 & 46 & 20 & 0 & 3 & & \\
\hline
\end{tabular}

Nota: i: Infante; j: Juvenil; P: Individuo periférico; RFEM1: Alto del Higo; RFEM2: Caras Pintadas. 
Cuadro 3. Densidades relativas de grupo por detección directa obtenidas para A. c. trabeata.

\begin{tabular}{|c|c|c|c|}
\hline Sitio de estudio & $\begin{array}{c}\text { Gira } 1 \\
\text { (grupo } / \mathbf{k m}^{2} \text { ) }\end{array}$ & $\begin{array}{c}\text { Gira } 2 \\
\text { (grupo } / \mathbf{k m}^{2} \text { ) }\end{array}$ & $\begin{array}{c}\text { Gira 3 } \\
\text { (grupo } / \mathbf{k m}^{2} \text { ) }\end{array}$ \\
\hline Altos del Higo & 0.002 & 1: $3 / 3$ días & 0.003 \\
\hline Tres Puntas & 0.003 & $1: 3 / 3$ dias & 0.001 \\
\hline Chupá & 0 & 1:3/3 días & 0.001 \\
\hline *Estivaná & 0 & 1:3/3 días & 0 \\
\hline
\end{tabular}

Nota: Los datos de densidad para Gira 2 fueron calculados en base a Poste de Escucha, se interpretan como 1 grupo detectado por esfuerzo de tres investigadores permaneciendo en el área por espacio de tres días. Algunos grupos detectados no siempre pertenecieron al área de estudio sino al adyacente, se colocó * para determinar que no pertenece al área de estudio, tomando en cuenta que los aullidos de los monos aulladores pueden ser escuchados a más de $1 \mathrm{~km}$ de distancia.

Cuadro 4. Índices de diversidad Alpha de los datos obtenidos por diferentes métodos en la Reserva Forestal El Montuoso y afluentes del Río la Villa.

\begin{tabular}{|c|c|c|c|}
\hline \multirow{3}{*}{ Diversidad Alpha } & \multicolumn{3}{|c|}{ Método } \\
\hline & \multicolumn{2}{|c|}{ RFEM } & \multirow{2}{*}{$\begin{array}{c}\text { Chupá } \\
\text { RN }\end{array}$} \\
\hline & FS & RN & \\
\hline $\mathbf{S}$ & 11 & 9 & 6 \\
\hline $\mathbf{n}$ & 79 & 45 & 22 \\
\hline D & 0.1687 & 0.3037 & 0.3967 \\
\hline 1-D & 0.8313 & 0.6963 & 0.6033 \\
\hline $\mathbf{H}^{\prime}$ & 2.008 & 1.609 & 1.26 \\
\hline Margalef & 2.289 & 2.102 & 1.618 \\
\hline Equitatibidad_J & 0.8373 & 0.7323 & 0.7034 \\
\hline Fisher_alpha & 3.473 & 3.383 & 2.718 \\
\hline Berger-Parker & 0.2911 & 0.5111 & 0.5909 \\
\hline Chao-1 & 11.5 & 10.5 & 7.5 \\
\hline
\end{tabular}

Nota: Fototrampeo en el sotobosque (FS), Redes de niebla (RN), Número de taxas identificadas (S), número de individuos (n), Dominancia (D), Simpson (1-D), Shannon (H'). 
Cuadro 5. Horas de esfuerzos de muestreo por método y visitas.

\begin{tabular}{|c|c|c|c|c|}
\hline Lugar & $\begin{array}{c}\text { 1 Gira } \\
21-25 \\
\text { de junio 2018 }\end{array}$ & $\begin{array}{c}\text { 2 Gira } \\
24-31 \text { de julio } \\
2018\end{array}$ & $\begin{array}{c}\text { 3 Gira } \\
\text { 31 agosto al 6 de } \\
\text { sept. 2018 }\end{array}$ & $\begin{array}{c}\text { Horas de } \\
\text { esfuerzo }\end{array}$ \\
\hline Transecto & 26 & 26 & 18 & 70 \\
Redes & 40 & 84 & 182 & 308 \\
SCo & Instalación & 2,160 & 3,600 & 5,700 \\
Horas totales & 66 & 2,270 & 3,800 & 6,136 \\
\hline
\end{tabular}

\section{DISCUSIÓN}

La riqueza de especies de mamíferos en zonas categorizadas como bosque continuo por la Reserva Forestal El Montuoso (RFEM) (Alto del Higo, Caras Pintadas y Tres Puntas) fue comparada estadísticamente con la de fragmento boscoso de río Estivaná-Gato y río Chupá ambos de la región de Macaracas, Herrera y Los Santos. Dado que hablamos de relación bosque donador-receptor (Wilson et al., 1997), la zona de bosque donador (RFEM) mantiene nueve Órdenes, 18 Familias, 37 Géneros y 44 especies de mamíferos. Se detectó cinco especies que no habían sido reportadas previamente en 2004 (Méndez-Carvajal et al., 2004), gracias al uso de las cámaras trampas (OCS) (Figura 3), por tanto, se incrementa la diversidad reportada en la RFEM a un 13\% (Méndez-Carvajal et al., 2004) tomando en cuenta que las especies anteriormente reportadas aun estarían allí, pero no fueron detectadas. Las especies adicionadas a la lista de mamíferos del RFEM son: Oecomys bicolor, Lyonicteris spurrelli, Caluromys derbianus, Tamandua mexicana y Puma yagouaroundi (Figura 2). Algunas especies no lograron reincidencia, sobre todo en cuanto a los Órdenes Lagomorpha, Artiodactyla, Chiroptera y Carnívora. La lluvia profusa en el muestreo impidió pernoctar en el área de Caras Pintadas de forma repetitiva, por tanto, perdimos horas de muestreo en una de las zonas de mayor cobertura boscosa, lugar donde se reportó Tyroptera tricolor y Bassaricyon gabbii (MéndezCarvajal et al., 2004) (Figura 1 y Cuadro 1). En total la diferencia de Órdenes de mamíferos presentes en ambos tipos de hábitats fue de un 50\%, 8 Órdenes para RFEM y 4 para bosques de galería, teniendo ambos al mono aullador $A$. c. trabeata como especie en común y a los murciélagos filostómidos. Esto implica que con el aumento de diversidad detectada para el bosque de la RFEM (bosque donador del río La Villa), los bosques de galería de los afluentes deberían mantener similar diversidad e ir disminuyendo de acuerdo al acercamiento con el área de mayor impacto antropogénico. Dado que el trabajo se relacionó con un indicador como el mono aullador $A$. c. trabeata, exploramos su relación ecológica con los demás mamíferos con los que comparte hábitat. Estudios similares sugieren un efecto positivo de regeneración a partir de zonas intervenidas por los trabajos de construcción del Canal de Panamá y la presencia del Monumento Natural de Isla Barro Colorado, donde A. palliata aequatorialis funge como especie clave en restauración boscosa de acuerdo a Milton et al., (2019).

Primates y otros mamíferos: Según la literatura, la presencia de primates del Género Alouatta, garantiza alta tendencia en regeneración de la vegetación nativa (Di-Fiore \& Campbell, 2007). De 
hecho, estudios señalan que, si un potrero o área de cultivo es abandonada para regeneración espontánea, es muy probable vuelva a servir para monos aulladores y estos a su vez mejorar su estructura boscosa (Quintana-Morales et al., 2011). De esta manera, la presencia de A. c. trabeata se relaciona a la conexión entre bosque donador RFEM y afluentes debido a su largo alcance de dispersión (8 a $40 \mathrm{Km}^{2}$ ), su capacidad de consumo de frutos (39\%), y su capacidad podadora de hojas verdes (45\%) (Glander et al., 2016). La actividad de A. c. trabeata en el dosel es de impacto grupal a multiescala pues se alimenta de yemas, hojas nuevas, flores, frutas y semillas, influyendo en la multiplicación de sitios de fructificación y abundancia de árboles, así, en el movimiento de otras especies de mamíferos dispersores de sotobosque como Cuniculus paca y Dasyprocta punctata (Méndez-Carvajal, 2012). Ambos roedores contribuyen a una cadena de eventos ecológicos por capacidad de romper semillas fuertes con su mandíbula (Cuniculus) (Smythe et al., 1982) y una olvidadiza capacidad de robo y enterramiento de semillas, que promueven la germinación al azar de ciertos árboles frutales (Dasyprocta y Sciurus) (Jansen et al., 2012; Emsens et al., 2013). Esta combinación de papeles ecológicos aumenta la posibilidad de regeneración de los bosques de galería de afluentes, mientras no haya impacto de cacería humana a gran escala. En nuestro trabajo las especies de mamíferos más activas en sotobosque fueron armadillos, ñeques, gatos solos y zarigüeyas a los que se les pudo calcular su actividad circadiana (Figura 3). Estas especies confirman su contribución como enterradores de semillas y polarizadores, escansorios, diurnos y nocturnos. Los grupos de A. c. trabeata han disminuido de seis a solo dos, uno en Caras Pintadas (RFEM) y uno en Tres Puntas (RFEM). La baja densidad de 0.04 ind $/ \mathrm{km}^{2}$ (antes 0.5 ind $/ \mathrm{km}^{2}$ ) puede ser normal para monos aulladores viviendo en zonas mayores a los $1000 \mathrm{~m}$ si se atiende a un factor de nutriente (Morales-Jiménez, 2002) aún por comprobar químicamente, sin embargo, a la altura de la RFEM las hojas y frutos deberían mantener buena calidad nutritiva y por tanto no debería haber densidad tan limitada. La densidad poblacional más pequeña para especies de similar característica grupal como A. c. trabeata ha sido la de A. pigra con 5 ind $/ \mathrm{km}^{2}$ en Tikal, Guatemala (Coehlo et al., 1976). En Isla Coiba se reporta densidad de $0.08 \mathrm{ind} / \mathrm{km}^{2}$ pero se mantiene la misma alerta y en Isla Coiba se considera la falta de frutos carnosos-azucarados este afectando su población (Méndez-Carvajal, 2012a). Consideramos en RFEM existe una presión negativa extra, quizá relacionada a la cacería o bien algún efecto parasítico. Se ha reportado presencia de Cuterebra baeri en Azuero (MéndezCarvajal, 2005) \& Milton (1996) sugiere la causa de muertes en los monos aulladores de Isla Barro Colorado en la zona del Canal de Panamá, se marca dependiendo de la edad del animal, su condición física y sus capacidades de reserva de grasa, sobre todo en el pico de la estación lluviosa por estrés alimenticio.

Los monos aulladores son entonces un buen indicador de condición saludable del bosque, tomando en cuenta que la detectabilidad es alta en muestreo o por su vocalización (Méndez-Carvajal \& SerioSilva, 2011). En promedios de número de individuos por grupos A. c. trabeata obtuvo en RFEM un valor de $\mathrm{x}=5$ (3-8; $\mathrm{n}=3$ ) individuos/grupo (Cuadro 2), más bajo en promedio a $A$. seniculus en Risaralda y Sucre, Colombia (x=6.1) (Morales-Jiménez, 2002; Ochoa et al., 2011), y similar a A. pigra en Hacienda Lakantún, Chiapas, México ( $\mathrm{x}=5.1$ ) (Estrada et al., 2004; Bonilla-Sánchez et al., 2011). No aun superando la baja de promedio grupal de A. c. coibensis en Isla Coiba, Panamá (x=4.3) (MéndezCarvajal, 2012a). La proporción de hembras por macho fue de 1:1.7, manteniendo su patrón unimacho con estructura similar y viable sin muestra de hacinamiento como la encontrada en los bosques fragmentados de Llano Grande de Ocú (Méndez-Carvajal, 2005). Esta estructura implica que ambos sexos se mantienen migrando en similar proporción, pero se deberá realizar más observaciones. Aunque este promedio coincide con su característica como especie no palliata (no multi-macho multihembra) (Whitehead, 1995; Méndez-Carvajal \& Serio-Silva, 2011), resalta en los resultados la baja de individuos juveniles e infantes, lo que lleva a pensar en un efecto externo que podría estar causando poco éxito de sobrevivencia. 
Especies de árboles reportadas para el RFEM y que son de importancia alimenticia para A. c. trabeata serían: Chrysophyllum cainito, Croton draco, Cecropia peltata, Byrsonima crassifolia, Lafoensia punicifolia, Inga marginata, Pachira sessilis, Hirtella racemosa, Ficus spp., Brosimum guianensis, Callophylum brasiliensis, Cedrela odorata, Cyathea petiolata, Myrcia gatunensis, de las cuales estas cuatro últimas pertenecen al listado de árboles en amenaza o vulnerables protegidas por ley (Garibaldi et al., 2004; Méndez-Carvajal, 2006; Delaney, 2008). Estas especies tendrían que ser encontradas y relacionadas en bosques de galería de los afluentes del río La Villa para hacer una relación de proporción de abundancia y relacionarlo con su dispersión. El mono cariblanco Cebus imitator es escaso en la RFEM y la densidad es baja en comparación con otras zonas montañosas protegidas, a pesar de la presencia de frutos de su preferencia como Inga spp (Jack, 2007). Por esta razón cuestionamos si los habitantes de Chepo de Las Minas, Sonadora y áreas aledañas se mantienen cazándolos como se reportó en Méndez-Carvajal et al. (2004). En Panamá, la presión de cacería en $C$. imitator aumenta al relacionarlo con un problema para las siembras de maíz y caña (Loría \& MéndezCarvajal, 2017). La extirpación del mono araña de Azuero A. g. azuerensis fue reportada por consumo por parte de pobladores de Sonadora hace unos 34 años (información de encuesta informal) (Méndez-Carvajal \& Ruiz-Bernard, 2009; Méndez-Carvajal et al., 2018). En este sentido, tenemos un reto al trabajar en la percepción comunitaria sobre estas especies antes de entablar cualquier proyecto de reintroducción de la especie A. g. azuerensis, tomando en cuenta que la RFEM posee buena fuente alimenticia para primates por su riqueza en árboles de las familias Annonaceae, Arecaceae, Cecropiaceae, Fabaceae, Lauraceae, Melastomataceae, Moraceae y Sapotaceae (Garibaldi et al., 2004; Glander et al., 2016). Además de los primates como indicadores, también evaluamos la relación de dispersores voladores, tales como murciélagos.

Mamíferos voladores: Las especies predominantes de murciélagos en la RFEM marcan el patrón con filostómidos como indicadores de bosque protegido, igual sugerido por Fleming et al., (1972), y la Carollia perspicillata se mantiene como líder con 15 individuos capturados, en comparación con el muestreo en 2004 en el cual se llegó a 16 individuos y de igual forma mantuvo la dominancia. Este patrón prevalece en zonas montañosas, concordando con otros estudios en la Serranía del Majé, Reserva Natural de Chucantí, Panamá con altura entre 800 a 1450 msnm (Walker et al., 2018; Méndez-Carvajal en prep.), Santuario Nacional Pampa Hermosa del Perú, donde el mismo Género Carollia (spp. C. brevicauda) predomina en un bosque de entre 1200 y 1600 msnm (Arias et al., 2016). En Parque Nacional Omar Torrijos y Parque Nacional Altos de Campana C. perspicillata fue reportado como especie predominante incluso con una amplia cobertura de muestreo y diversidad de murciélagos para ambas regiones (Araúz, 2002; 2006). Mientras que en las zonas de bosque de galería de los afluentes río La Villa la presencia de murciélagos hematófagos como el Desmodus rotundus fue mayor. Otras especies mayormente insectívoras fueron encontradas en Chupá debido quizá a la proliferación de ganadería y a los espacios abiertos producto de agricultura que fragmentan el paisaje boscoso, similar a García-García y Santos-Moreno (2014) en su trabajo de ensamblaje de murciélagos en fragmentos y bosques maduros en Los Chimalapas de Oaxaca, México. Aun así, un murciélago frugívoro dominó la abundancia relativa de especies de fragmentos, el Artibeus jamaicensis en los bosques de ríos afluentes podrían mantener presencia alta debido a la floración de especies de los géneros Anacardium, Cecropia, Ficus, Spondias y Manguifera presentes en las orillas de ríos y cercas vivas de los potreros, representando una especie de gran valor para la proliferación de semillas y continuidad del bosque por oportunismo ecológico (Giannini \& Kalko, 2004). Estivaná, sin embargo, reportó un murciélago común en diferentes hábitats fragmentados en Azuero y está relacionado a una alimentación de néctar y polen; Glossophaga soricina, forrajea repetitivamente incluso en árboles no adaptados a polinizadores nocturnos y voladores, esto indica su amplio papel de polinizador aún en zonas intervenidas, similar a otras especies arborícolas como Didelphis marsupialis, Caluromys derbianus y ratones como Oecomys bicolor (Keys et al., 2012). Estos murciélagos pueden usar estructuras como puentes, cavernas o huecos de árboles por lo que, en lugares con actividad 
antropogénica, son comúnmente capturados si hay bosque cerca (Barquez et al., 2015). Se necesitará realizar otro muestreo en estación seca para complementar data si se quisiera completar la lista de mamíferos actual de la RFEM y comprender la ecología de los murciélagos estacionales. Los indiscutibles indicadores de bosque diverso y protegido son C. perspicillata para bosque maduro protegido, y Desmodus rotundus para bosque alterado, y su abundancia podría representar problemas como vector de zoonosis (Greenhal, 1968). En general la familia Phyllostomidae es de las más diversas en preferencia alimenticia y monitorear su abundancia relativa a través del tiempo podría marcar patrones de degradación de hábitat (Heithaus et al., 1975; Kalko et al 2001; Araúz, 2006).

Al aplicar los índices de diversidad ecológica de Shannon-Weinner se obtuvo claramente mayor diversidad en RFEM, respecto al bosque de galería (Cuadro 3), este índice refleja la importancia de la conectividad de los bosques de RFEM con los bosques de galería a manera de ensamblaje ecológico (Durán \& Pérez, 2015). A nivel de los demás grupos de mamíferos obtenidos se notó ausencia de especies comunes en el 2004 como venados Mazama temama, Odocoileus virginianus, y carnívoros grandes como Panthera onca. La RFEM solo reporta un incidente con un jaguar en 2012 cuando una hembra con su cría fue avistada por una mujer que iba a caballo, aunado al caso del jaguar baleado en Chirote-Pitaloza (Los Pozos, provincia de Herrera). La ausencia de jaguares o pumas amerita atención y monitoreo para complementar datos de percepción humana hacia el felino y reforzar la protección del mismo, ya que es una de las especies más perseguida por el hombre y en Azuero casi extirpada (Moreno et al., 2016; Fort et al., 2017).

\section{CONCLUSIÓN}

Nuestros resultados demuestran que la evaluación de una especie indicadora como el mono aullador, es viable, por ser una especie que es diurna, visible al observador y detectable, sin embargo, se debe tener en cuenta el uso de la especie por el ser humano en el entorno de estudio. Los monitoreos de esta especie de manera anual y a largo plazo deben ser implementados en zonas de alto impacto y sus densidades calculadas para evaluar de mejor forma la red presencial de especies de árboles y otros mamíferos en el área. Aunque las especies de mamíferos aumentaron el listado por detección en RFEM, esto se debió mayormente a la implementación de nuevas técnicas de detección y no de inclusión por uso de tierra y cobertura boscosa. Los monos aulladores demuestran que alguna variación en el entorno está causando una baja poblacional en los monos aulladores de RFEM y esta situación pone en evidencia una baja detección de animales de sotobosque en las zonas adyacentes y bosques de galería de ríos afluentes. Por esta razón, una acción rápida para ayudar al ecosistema a mantener la cobertura boscosa sería la reforestación con especies de árboles nativos y el uso de cercas vivas tradicionales con especies como Anacardium excelsum, Spondias mombin, que componen parte de la dieta del mono aullador en los bosques de ríos afluentes a río La Villa. Otra recomendación sería evitar el uso de herbicidas que podrían envenenar a especies que ayudan a mantener la regeneración rápida de los árboles nativos, tales como Artibeus jamaicensis. Este estudio ha dado un perfil de la población del mono aullador de Azuero en una de las pocas zonas montañosas protegidas de la región, en donde mantiene sus condiciones naturales sin hacinamiento, el proyecto sugiere utilizar a estas especies como indicadoras para potenciales de regeneración en zonas fragmentadas.

Conservación: El Ministerio de Ambiente por medio de su Proyecto Pro-Cuencas, el Centro Regional Universitario de Coclé (CRU-Coclé), la Universidad de Panamá y FCPP, implementaron una reforestación en los predios de río Chupá de unos 1,500 plantones y 100 semillas de especies nativas como: Cocobolo (Dalbergia retusa), María (Calophyllum brasiliense), Macano (Diphysa americana), Espavé (Anacardium excelsum), Guachapalí (Samanea saman), Malagueto (Xylopia aromatica) y se organizó instrucción de reforestación por parte de Minera Panamá S.A., como colaboradores y facilitadores a estudiantes de CRU Coclé. FCPP dió instrucción a guardaparques de la RFEM en temas de detección de mamíferos utilizando el Sistema de Cámaras Orión (SCO). 


\section{AGRADECIMIENTOS}

Agradecemos a la administración del Centro Regional Universitario de Coclé, por el apoyo brindado en actividad de reforestación; a los estudiantes del cuarto año de la Licenciatura en Biología y Turismo Ecológico del CRU Coclé por el apoyo en la jornada de reforestación en Chupá y Estivaná; a Minera Panamá S.A., por la asesoría técnica y donación de semillas utilizadas en la reforestación; a MiAmbiente y proyecto PROCUENCAS: Ana Cristina Nicosia, Mercedes Ochoa, Eric Nuñez, José Betancourt, y Marcos Salabarría, por hacer posible este trabajo, donarnos los plantones utilizados en la reforestación, en especial a los guardaparques de la RFEM: Reyes Ojo Noriega, Marcos Saavedra, Luis Atencio, Didacio Ramos, Ladislao Marín, y su director Ítalo Ramírez. Agradecemos a Yostin Añino y al equipo editorial de Mesoamericana por la edición y formato del manuscrito.

\section{CONFLICTO DE INTERESES}

Los autores presentes declaran que no existe intereses de conflicto.

\section{BIBLIOGRAFÍA}

ARANDA, J. M. 2000. Huellas y otros rastros de los mamíferos grandes y medianos de México. Comisión Nacional para el Conocimiento y uso de la Biodiversidad. Primera edición. Instituto de Ecología. Xalapa, Ver. México, pp 212.

ARAÚZ G, J. 2002. Los murciélagos del sendero Panamá, Parque Nacional Altos de Campana. Tecnociencia, $4(2): 35-48$.

ARAÚZ G, J. 2006. Riqueza de especies y abundancia de murciélagos en algunas localidades de Panamá Central. Tecnociencia, 8(2):171-190.

ARROYO-RODRÍGUEZ, V., MANDUJANO, S., \& CUENDE-FANTON, C. 2005. Ocupación de parches de selva por monos aulladores Alouatta palliata mexicana en tres paisajes con diferente grado de fragmentación en Los Tuxtlas, México. Universidad y Ciencia, (II), 23-34.

ARCIA, D., CABALLERO, A. \& GARCÍA, J. 2004. Medio físico de la reserva forestal El Montuoso In: Diversidad Biológica y Servicios Ambientales de los Fragmentos de Bosques en la Reserva Forestal El Montuoso, C. Garibaldi (ed.) Universal Books, Panamá, pp.21-27.

ARIAS E., V. PACHECo, K. CERVANTES, A. AGUILAR \& J. ÁlVAREZ. 2016. Diversidad y composición de murciélagos en los bosques montanos del Santuario. Nacional Pampa Hermosa, Junín. Perú.Revista Peruana de Biología. 23(2): 103 - 116.

BONILLA-SÁNCHEZ, Y., POZO-MONTUY, G. \& SERIO-SILVA, J. C. 2011. Demografía y Evaluación del hábitat del mono aullador negro (Alouatta pigra) en Playas de Catazajá Chiapas. En: Perspectivas en Primatología Mexicana, L. M. Gama-Campillo, G. Pozo-Montuy, W. M. Contreras-Sánchez, and S. L. Arriaga-Weiss (eds.). Universidad Juárez Autónoma de Tabasco, México. 189-206 pp.

CHAO, A. 1987. Estimating the population size for capture-recapture data with unequal catchability. Biometrics, 43: 783-791.

COEHLO, A. M., BRAMBBLETT, JR., C.A., QUICK, L. B. \& BRAMBLETT, S. S. 1976. Resource availability and population density in primates: a socio-bioenergetic analysis of energy budget of Guatemalan howler and spider monkeys. Primates, 17:63-80. 
COLWELL, R. K. \& CODDINGTON, J. A. 1994. Estimating terrestrial biodiversity through extrapolation. Philosophical Transactions of the Royal Society, 345:101-118.

CORREA-GÓMEZ, D. F., \& STEVENSON, P. R. 2010. Structure and diversity of riparian forests in a seasonal savanna of the Llanos Orientales Colombianos (Tomo Grande Reserva, Vichada). Orinoquia, 14sup (1): 31-48.

COWLISHAW, G., \& DUNBAR, R. I. 2000. Primate conservation biology. University of Chicago Press.

CUARÓN, A. D., MORALES, A., SHEDDEN, A., RODRÍGUEZ-LUNA, E. \& DE GRAMMONT, P. C. 2008. Ateles geoffroyi ssp. azu- erensis. In: IUCN Red List of Threatened Species. Ver- sion 2009.1. Website: www.iucnredlist.org. Accessed: 2 November 2009.

DA SILVA, A.G., GAONA 0. \& MEDELLÍN, R.A. 2008. Diet and trophic structure in a community of fruit-eating bats in Lacandon Forest, México. Journal of Mammalogy, 89(1):43-49.

DELANEY, K. 2008. The Azuero howler monkey (Alouatta coibensis trabeata), use of natural resources in the El Montuoso Forest Reserve in Herrera province, Panama.

DI FIORE, A. \& CAMPBELL, C.J. 2007. The Atelines: Variation in Ecology, Behaviour, and Social Organization. En: Campbell C. J., Fuentes, A., Mackinnon, G., Panger, M., Bearder, S. (eds). Primates in Perespective. Oxford University Press, New York, pp. 155-185.

DURÁN, A. A. \& PÉREZ, S. C. 2015. Ensamblaje de murciélagos (Mammalia: Chiroptera) en dos zonas del departamento de Sucre, Colombia. Acta Zoológica Mexicana, 31(3): 358-366.

EMSENS, W. J., SUSELBEEK, L., HIRSCH, B.T., KAYS, R., WINKELHAGEN, A. J. S. \& JANSEN, P. A. 2013. Effects of food availability on space and refuge Use by a Neotropical Scatterhoarding Rodent. Biotropica, 45(1): 88-93.

ESTRADA, A., VAN-BELLE, S. \& GARCIA DEL VALLE, Y. 2004. A survey of black howler (Alouatta pigra) and spider (Ateles geoffroyi) monkeys along the Río Lacantún, Chiapas, Mexico. Neotrop. Primates, 12(2): 70-75.

FLEMING, T., HOOPER, E. \& D. WILSON. 1972. Three Central American bat communities: structure, reproductive cycles, and movement patterns. Ecology, 53: 555-569.

GARCÍA-GARCÍA, J. L., \& SANTOS-MORENO, A. 2014. Variación estacional en la diversidad y composición de ensambles de murciélagos filostómidos en bosques continuos y fragmentados en Los Chimalapas, Oaxaca, México. Revista mexicana de biodiversidad, 85(1): 228-241.

GARIBALDI, C., AGUILAR, S., ARCIA, D. \& TORRES, N. 2004. La vegetación arbórea en los bosques fragmentados de la reserva forestal El Montuoso. En: C. Garibaldi (ed.). Diversidad Biológica y Servicios Ambientales de los Fragmentes de Bosques en la Reserva Forestal El Montuoso, Universal Books, Panamá, 39$63 \mathrm{pp}$.

GAULIN, S. J., KNIGHT, D. H., \& GAULIN, C. K. 1980. Local variance in Alouatta group size and food availability on Barro Colorado Island. Biotropica, 12(2): 137-143.

GIANNINI, N. P. \& KALKO, E. K. 2004. Trophic structure in a large assemblage of phyllostomid bats in Panama. Oikos, 105(2): 209-220.

GLANDER, K. E., ESTRADA, A., MANDUJANO, S. \& MÉNDEZ-CARVAJAL, P. 2016. Mantled howler monkey Alouatta palliata. En: Noel Rowe \& Marc Myers (eds). All the world primates. Pogonias Press, Charlestown, Rhode Island, USA, pp. 250-254. 
GLANZ, W. 1992. Fauna de mamíferos terrestres de la isla Barro Colorado: Censos y cambios a largo plazo. En: E. Leigh et al. (Eds). Ecología de un Bosque Tropical. Presencia LTDA, Colombia. Smithsonian Tropical Research Institue, Balboa, República de Panamá.

GREENHAL, A. M. 1968. Vampire bats and the paralytic rabies. Problems and complications in the control of vampire bats. Union Interconservacion Rec. Nat...Conf. Lat. Am. Reg. Con. Rec. Na. Re. San Carlos de Bariloche. B5(1):1-9.

HANDLEY, C. 0. 1981. Key to the bats of the lowlands of Panama. U.S. Natl. Mus. Washington, D.C. 17 p.

HEITHAUS, E. R., FLEMING, T. H., \& OPLER, P. A. 1975. Foraging patterns and resource utilization in seven species of bats in a seasonal tropical forest. Ecology, 56(4): 841-854.

HELTSHE, J. \& FORRESTER, N. E. 1983. Estimating species richness using the jackknife procedure. Biometrics, $39(1): 1-11$

JACK, K.M. 2007. The Cebines: Toward an Explanation of Variable Social Structure. En:, Campbell CJ, Fuentes A, Mackinnon G, Panger M, Bearder S (eds). Primates in Perespective. Oxford University Press, New York. 107$123 \mathrm{pp}$.

JANSEN, P. A., HIRSCH, B. T., EMSENS, W. J., ZAMORA-GUTIERREZ, V., WIKELSKI, M., \& KAYS, R. 2012. Thieving rodents as substitute dispersers of megafaunal seeds. Proceedings of the National Academy of Sciences, 109(31): 12610-12615.

JOST, L., CHAO, A. \& CHAZDON, R. L. 2011. Compositional similarity and b (beta) diversity. In: Anne E. Magurran \& Brian J. (Ed.). Biological Diversity: frontiers in measurement and assessment. McGill, Oxford University Press Inc, New York, USA: 66-84 pp.

KALKO, E. K., \& HANDLEY, C. 0. 2001. Neotropical bats in the canopy: diversity, community structure, and implications for conservation. Plant ecology, 153(1-2): 319-333.

KAYS, R. 2012. Visita de Animales y Polinización de Árboles de Balso (Ochroma pyramidale) en Panamá. Mesoamericana, 16(3): 56-70.

LORÍA, L. I., \& MÉNDEZ CARVAJAL, P. G. 2017. Uso de habitat y patrón de actividad del mono cariblanco (Cebus imitator) en un agroecosistema cafetalero en la Provincia de Chiriquí, Panamá. Tecnociencia, 19(1): 61-78.

MAGURRAN, A. E. 2007. Species abundance distributions over time. Ecology letters, 10: 347-354.

MASSEY, A. 1987. A population survey of Alouatta palliata, Cebus capucinus and Ateles geoffroyi at Palo Verde, Costa Rica. Revista de Biologia Tropical, 35(2): 345-347.

MASÍS, F., VALDEZ, J., COTO, T., \& LEÓN, S. 2008. Residuos de agroquímicos en sedimentos de ríos, Poás, Costa Rica. Agronomía Costarricense, 32(1): 113-123.

MÉNDEZ, E. 1993. Los Roedores de Panamá. Impresora Pacífico S.A.372 pp.

MÉNDEZ-CARVAJAL, P. G. 2005. Population Survey of the Azuero Howler Monkey (Alouatta palliata trabeata) in Herrera Province, Republic of Panama. Neotropical Primates, 13(3): 1-6.

MÉNDEZ-CARVAJAL, P. G. 2012. Population study of Coiba howler monkeys (Alouatta coibensis coibensis) and Coiba capuchin monkeys (Cebus capucinus imitator), Coiba Island National Park, Republic of Panama. Journal of primatology, 1(2): 104. 
MÉNDEZ-CARVAJAL, P. G. 2013. Population size, distribution and conservation status of howler monkeys (Alouatta coibensis trabeata) and spider monkeys (Ateles geoffroyi azuerensis) on the Azuero Peninsula, Panama. Primate Conservation, 26(1): 3-15.

MÉNDEZ-CARVAJAL, P.G. 2014. The Orion Camera System, a new method for deploying camera traps in tree canopy to study arboreal primates and other mammals: a case study in Panama. Mesoamericana, 18(1): 9-23.

MÉNDEZ-CARVAJAL, P. G., SANTAMARÍA, E. \& GARIBALDI, C. 2004. Riqueza y diversidad de mamíferos silvestres en los remanentes de bosques de la Reserva Forestal El Montuoso. In: Garibaldi, C. (ed). Diversidad Biológica y Servicios Ambientales de los Fragmentos de Bosques en la Reserva Forestal El Montuoso, Panamá. Universal Books, Panamá, República de Panamá. 161-171 pp.

MÉNDEZ-CARVAJAL, P. G. \& RUIZ-BERNARD, I. 2009. Estudio poblacional del mono araña de Azuero (Ateles geoffroyi azuerensis), Península de Azuero, Panamá. Tecnociencia, 11(1): 29-44.

MÉNDEZ-CARVAJAL, P. G., RUIZ-BERNARD, I., BERGUIDO, G. \& AIZPRÚA, R. 2010. Estudio poblacional de primates en la Reserva Chucantí, Provincia del Darién, República de Panamá. Congreso Mesoamericano de Biología y Conservación, p.48. San José, Costa Rica, 8-12 November 2010. Abstract.

MÉNDEZ-CARVAJAL, P. G. \& SERIO-SILVA, J.C. 2011. Daybreak chorus and bark analysis for two species of howler mon- keys Alouatta coibensis and Alouatta palliata: Atelidae, in Republic of Panama. En: L. M. GamaCampillo, G. Pozo-Montuy, W. M. Contreras-Sánchez, and S. L. Arriaga-Weiss (eds). Perspectivas en Primatología Mexicana. Universidad Juárez Autónoma de Tabasco, México. 167-184 pp.

MÉNDEZ-CARVAJAL, P.G., RODRÍGUEZ-MENJÍVAR, M., POZO-MONTUY, G., CHAVES, O.M., PONCE, G., RODRÍGUEZ-BEITIA, B.A. \& PORTILLO-REYES, H. 2018. Central American Spider Monkey Ateles geoffroyi Kuhl, 1820: Mexico, Guatemala, Nicaragua, Honduras, El Salvador, Costa Rica, Panama. 2016. In: C. Schwitzer, R.A. Mittermeier, A.B. Rylands, F. Chiozza, E.A. Williamson, E.J. Macfie, J. Wallis and A. Cotton (eds.). Primates in Peril: The World's 25 Most Endangered Primates 2016-2018.IUCN SSC Primate Specialist Group (PSG), International Primatological Society (IPS), Conservation International (CI), and Bristol Zoological Society, Arlington, VA. 91-95 pp.

MEYER, N., ESSER, H. J., MORENO, R., VAN LANGEVELDE, F., LIEFTING, Y., OLLER, D., VOGELS, C. B. F., CARVER, A.D., NIELSEN, C. K. \& JANSEN, P. A. 2015. "An assessment of the terrestrial mammal communities in forests of Central Panama, using camera-trap surveys." Journal for Nature Conservation, 26: 28-35.

MILTON, K. 1996. Effects of bot fly (Alouattamyia baeri) parasitism on a free-ranging howler monkey (Alouatta palliata) population in Panama. Journal of Zoology, 239(1): 39-63.

MORALES-JIMÉnEZ, A. L. 2002. Densidad de los monos aulladores (Alouatta seniculus) en un bosque subandino, Risaralda, Colombia. Neotropical Primates, 10(3): 141-144.

MORENO, R., A. BUSTAMANTE, P.G. MÉNDEZ-CARVAJAL \& MORENO J. 2016. Jaguares (Panthera onca) en Panamá: Estado actual y conservación. In: R. A. Medellín, J. A. de la Torre, C. Chávez, H. Zarza, and G. Ceballos, editors.El jaguar en el Siglo XXI: La perspectiva Continental. Fondo de Cultura Económica, Universidad Nacional Autónoma de México, Ciudad de México.

MOSQUERA-MUÑOZ, D. M., CORREDOR, G., CARDONA, P., \& ARMBRECHT, I. 2014. Fototrampeo de aves caminadoras y mamíferos asociados en el piedemonte de Farallones de Cali. Bol. Cient. Mus. Hist. Nat. U. de Caldas, 18(2): 144-156.

PALIZA-GARCíA, E. 2018. Pertinencia de los mamíferos como indicadores de diversidad biológica en las evaluaciones ambientales. La Molina, Facultad de Ciencia, Universidad Nacional Agraria. Lima, Perú. Tesis de Licenciatura. 
REID F.A. 1997. A field guide to the mammals of Central America and Southeast Mexico. Oxford University Press. New York, USA, 334 pp.

RODRÍGUEZ SOSA, J. L., PUIG PÉREZ, A., \& LEYVA MAGAÑA, C. P. 2018. Caracterización estructural del bosque de galería de la Estación Experimental Agroforestal de Guisa. Revista Cubana de Ciencias Forestales, 6(1): 45-57.

ROSALES-MEDA. 2006. Población de mono congo en isla San Lucas. Revista de Ciencias Ambientales, 32(1): 2830.

ROSS, C. \& REEVE, N. 2011. Survey and census methods: population distribution and density [En línea]. En: J. Setchell y D. Curtis, eds., Field and laboratory methods in primatology. Disponible en: https://books.google.com.pa. [Consultado 2 jul 2018].

RUMIZ, D. I. 2010. Roles ecológicos de los mamíferos medianos y grandes. Distribución, ecología y conservación de los mamíferos medianos y grandes de Bolivia. 53-73 pp.

SMYTHE, N., GLANZ, W.E. \& LEIGH, E.G. JR. 1982. Population regulation in some terrestrial frugivores. En: E.G. Leigh, A.S. Rand, and D.M. Wind- sor (Eds.). The ecology of a tropical forest: seasonal rhythms and long-term changes. Smithsonian Institution press, Washington DC., pp. 227-238.

WALKER, M. J., DORRESTEIN, A., CAMACHO, J. J., MECKLER, L. A., SILAS, K.A., HILLER, T. \& HAELEWATERS, D. 2018. A tripartite survey of hyperparasitic fungi associated with ectoparasitic flies on bats (Mammalia: Chiroptera) in a neotropical cloud forest in Panama. Parasite, 25:19.

WILSON, D.E., ASCORRA, C.F. \& SOLARI., E. 1996. Bats as indicators of habitat disturbance. P.p. 613-624. En: D.E. Wilson \& A. Sandoval (Eds.) Manu: The Biodiversity of Southeastern Perú. Smith. Instit. Nat. Hist. Nat. Mus. \& Editorial Horizonte, Lima Perú. 679 pp.

WHITTAKER, R.H. 1960. Vegetation of the Siskiyou mountains, Oregon and California. Ecological Monographs, 30:279-338.

ZUNINO, G. E., KOWALEWSKI, M. M., OKLANDER, L. I., \& GONZÁLEZ, V. 2007. Habitat fragmentation and population size of the black and gold howler monkey (Alouatta caraya) in a semideciduous forest in northern Argentina. American Journal of Primatology, 69(9): 966-975.

Citar como: Méndez-Carvajal, P., Gómez de Huertas, I., Gutiérrez-Pineda, K., Moreno, R., Peñafiel, M., Girón-Rengifo, A., Méndez-Carvajal, E., González-Hernández, P. \& Ortiz, A. 2020. Potencial regenerativo de bosques de galería en base a diversidad y abundancia de mamíferos en la Reserva Forestal El Montuoso y afluentes del río La Villa, Herrera, Panamá. Mesoamericana 24(1): 58-76. 


\section{ANEXOS}

Figura 2. Actividad circadiana de algunos mamíferos terrestres detectados por Fototrampeo en la Reserva Forestal El Montuoso.

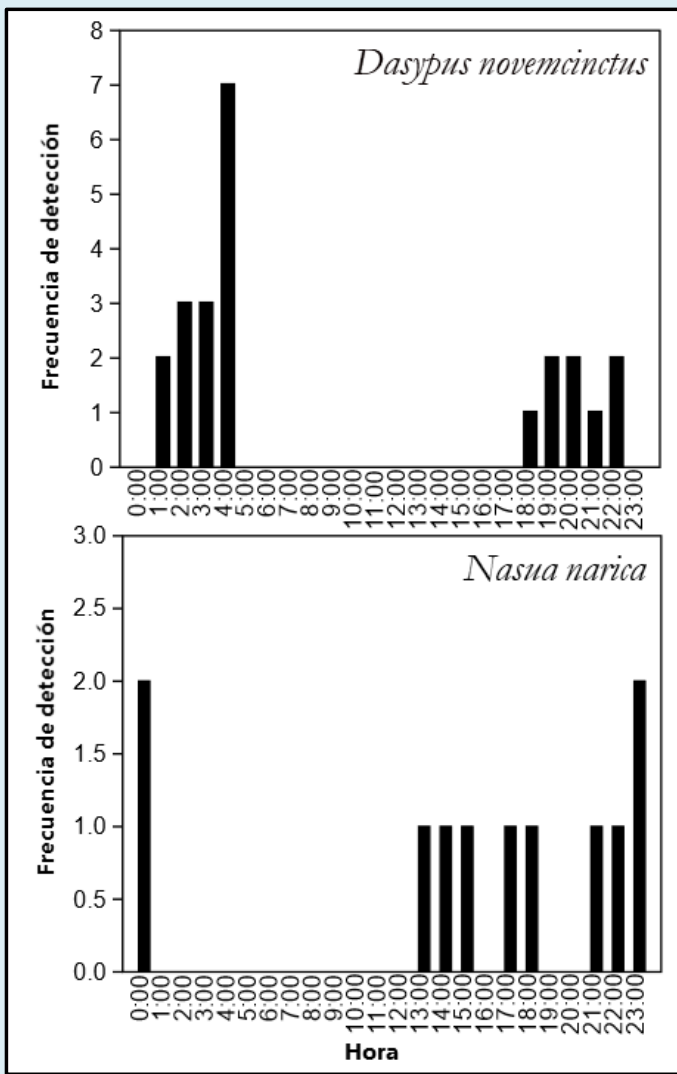

Hora

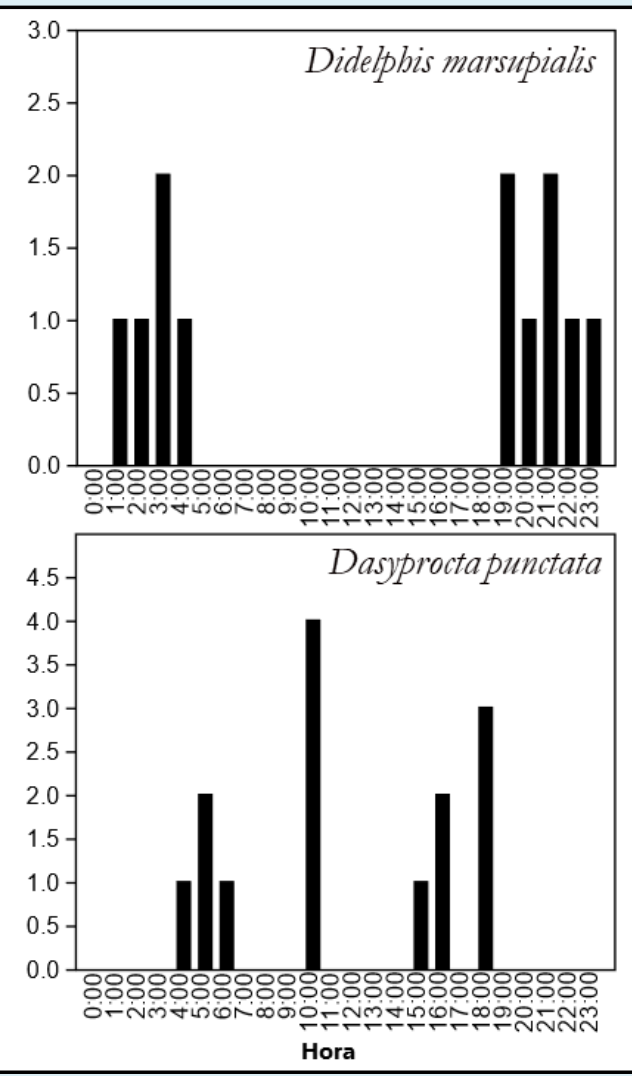

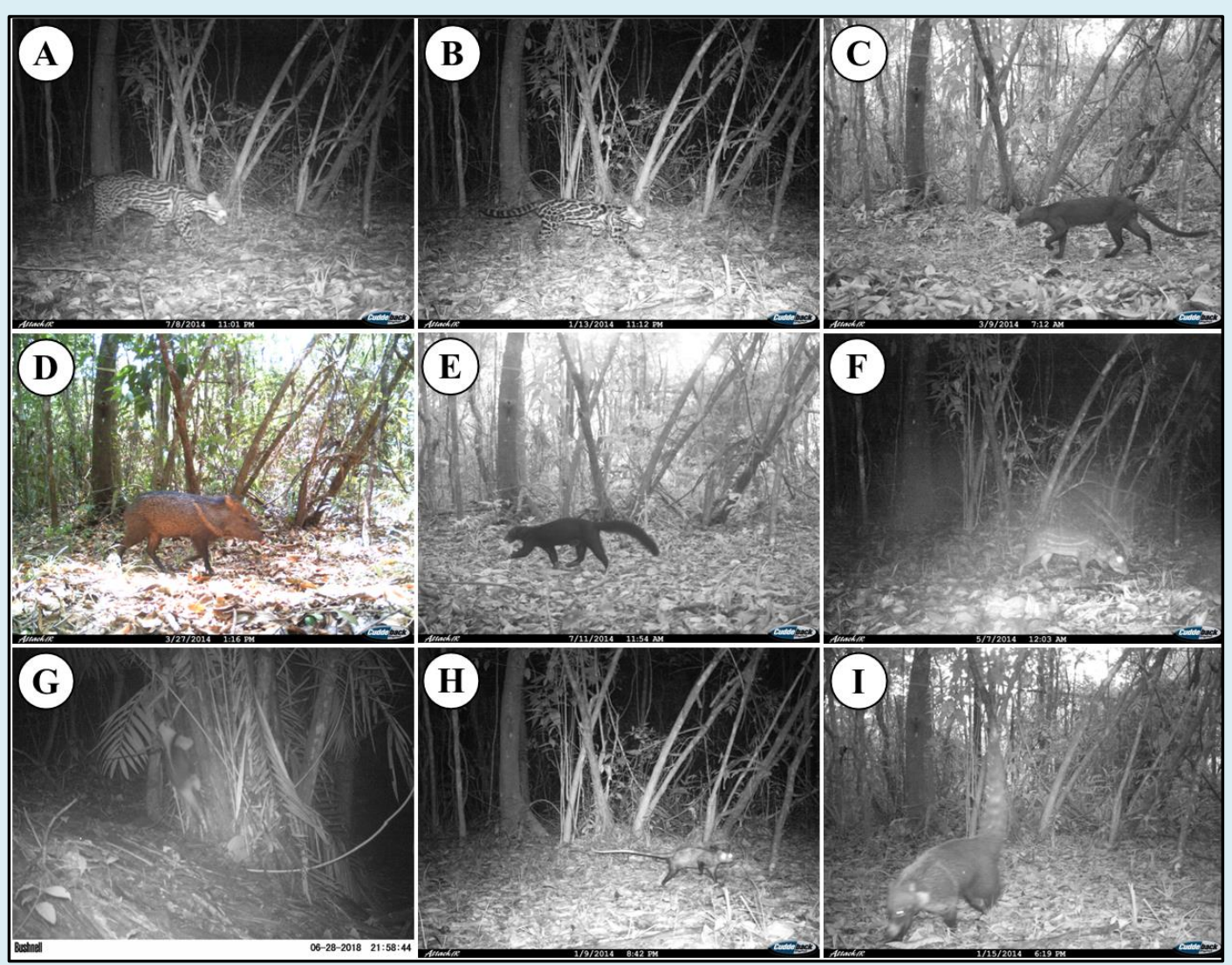

Figura 3. Mamíferos detectados por fototrampeo. A. Leopardus pardalis, B. Leopardus wiedii, C. Puma yagouaroundi, D. Pecari tajacu, E. Eira barbara F. Cuniculus paca, G. Tamandua mexicana, H. Didelphis marsupialis, I. Nasua narica. 


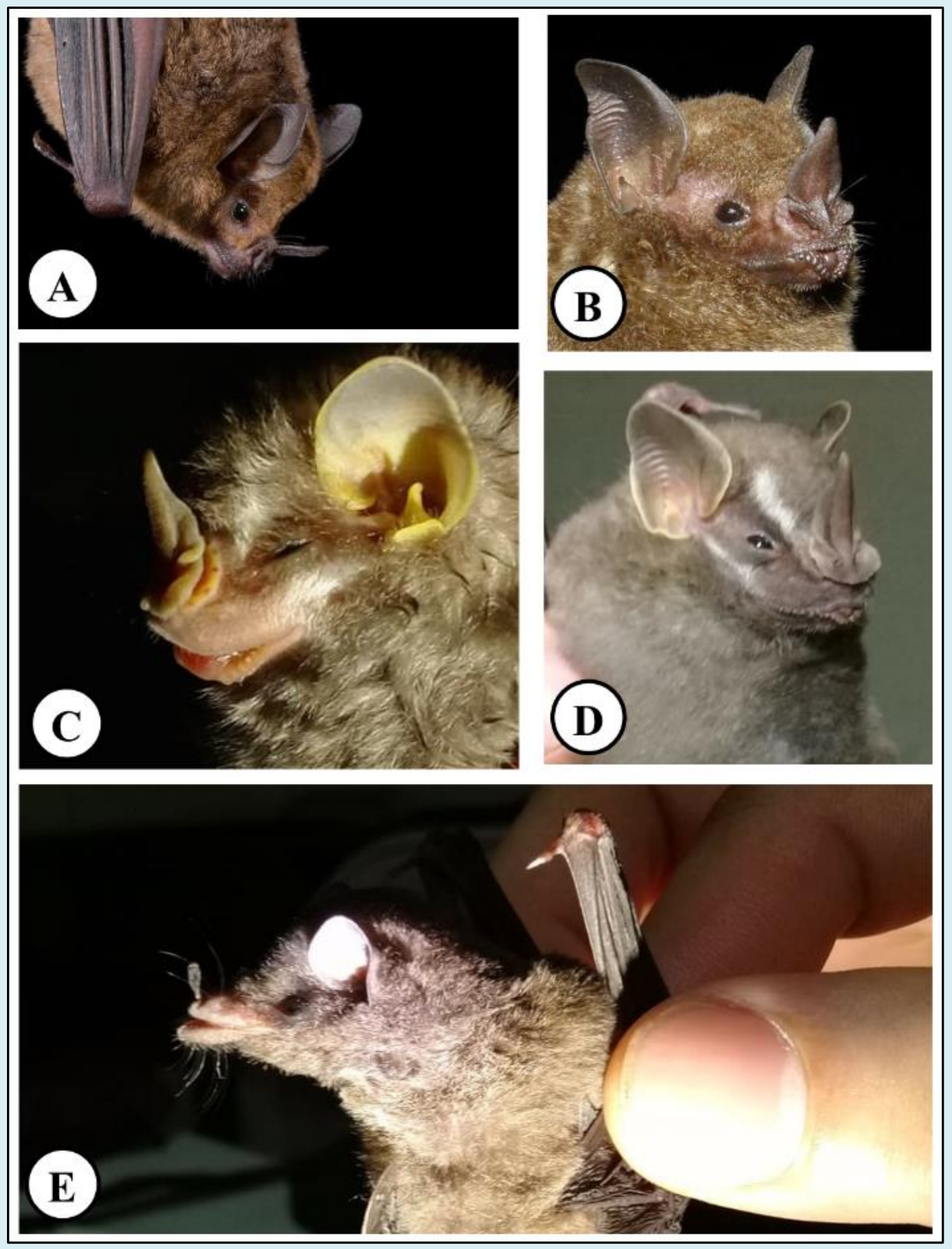

Figura 4. Especies de murciélagos atrapados en redes de niebla y posteriormente liberados. A. Carollia castanea, B. Carollia perspicillata, C. Vampyressa pusilla*, D. Artibeus phaeotis, E. Lionycteris spurrelli*. Especies nuevas para RFEM (*). 